anaplastic large-cell lymphoma in adults. It is currently awaiting conditional marketing authorization for adults in Europe. A Phase I/II study in paediatrics is at the moment recruiting. Brentuximab vedotin is administered every three weeks at $1.8 \mathrm{mg} / \mathrm{kg}$ (half-life ranges from 4 to 6 days and steady-state was achieved in 21 days for the ADC). Administration is possible in France, after the ANSM granted it temporary authorization on a named patient basis.

An 8-year-old male child, with a diagnosis of anaplastic large-cell lymphoma, was treated according to the ALCL99 protocol. Two months after diagnosis the tumour grew under this first-line chemotherapy. A multidisciplinary group decided to start brentuximab vedotin treatment. A total of 5 courses spaced 3-weekly were scheduled combined with chemotherapy. Signs of the tumour disappeared, thorax imaging normalised, fever and pulmonary and mediastinum adenopathies decreased.

Conclusions After the 4th dose of brentuximab vedotin, the treatment was well tolerated by the patient and the tumour regressed. Among adults, the median response is about 12 months. Thus, confirmation of efficacy still has to be evaluated. Further studies are required to establish the efficacy and safety profile in the paediatric population.

No conflict of interest.

\section{DGI-079 VALPROIC ACID AND BEHAVIOUR DISORDERS: OBSERVATION OF EFFICIENCY AND TOXICITY IN A COGNITIVE-BEHAVIORAL UNIT}

doi:10.1136/ejhpharm-2013-000276.345

${ }^{1} \mathrm{C}$ Rouzaud Laborde, ${ }^{1} \mathrm{~A}$ Rigal, ${ }^{1} \mathrm{~F}$ Boye, ${ }^{2} \mathrm{~B}$ Vellas, 'P Cestac. 'Toulouse University Hospital, Pharmacy, Toulouse, France; ${ }^{2}$ Toulouse University Hospital, Gerontology, Toulouse, France

Background In order to limit neuroleptic use in the elderly, because of cardiovascular events, specialists in charge of behaviour disorders don't have many therapeutic options in cognitive-behavioural units (CBU).

Purpose Valproic acid (VPA) is an anticonvulsant and/or a mood stabiliser that can be used in a behavioural way in CBU. One side effect of VPA is hyperammonaemia, which can lead to sedation and changes in behaviour or personality.

Materials and Methods Inclusion criteria were opposition, agitation, aggressiveness or impulsiveness. Ammoniemia levels were assessed before starting the VPA, between 2 and 4 days and after 5 days with VPA. For each person included, Cockroft's creatinine clearance, medical background and neuroleptic co-prescriptions were identified. Results are presented with mean \pm SEM.

Results The population was defined by an average age of $79.3 \mathrm{y}$ \pm 1.74 , a sex ratio of 15 men for 6 women; a creatinine clearance of $65.4 \mathrm{~mL} / \mathrm{min} \pm 8.9$, no patients had liver troubles or a history of epilepsy. 21 patients received VPA in the CBU, for at least one of the following indications: opposition $(n=9)$, agitation ( $n=13)$, aggressiveness $(n=16)$ or impulsiveness $(n=6) .9 / 21$ patients came out of the CBU with VPA (42.85\%), 13/21 without VPA (61.9\%), $5 / 21$ with a neuroleptic $(23.8 \%)$ and $8 / 21$ without VPA or a neuroleptic $(38.1 \%)$. Ammoniemia rates at D-1, between D2 and D4 and after D5 were respectively $47.47 \mu \mathrm{M} \pm 3.71,51.4 \mu \mathrm{M} \pm 6.43$ and $63.76 \mu \mathrm{M} \pm 4.95$. Response rate to VPA was $55 \%$ (5/9 patients) for opposition, $37.5 \%$ (6/16) for aggressiveness, $38 \%$ (5/13) for agitation and $66.6 \%(4 / 6)$ for impulsiveness.

Conclusions Those results show that only one of every two patients with VPA were responders, and average ammoniemia increases during treatment. However, $100 \%$ of patients going out with VPA didn't have any neuroleptics and for 33\%, VPA contributed to stopping neuroleptics.

No conflict of interest.

\section{Pharmacotherapy: pharmacokinetics and pharmacodynamics (including: ADE, TDM, DUE)}

\section{PHC-001 AMIKACIN DOSING TO TREAT RESPIRATORY TRACT INFECTIONS ACCORDING TO PATIENT'S BODY MASS INDEX}

doi:10.1136/ejhpharm-2013-000276.346

M Barrantes-González, M Marín-Casino, B Lopez, S Ortonobes, E Salas, S Grau. Hospital de Mar, Pharmacy, Barcelona, Spain

Background Body mass index (BMI) is a factor related to the disposition of aminoglycosides (AMG). Dosage is based on total body weight (TBW) or adjusted body weight (ABW) according to patients' BMI.

Purpose To assess if the amikacin dosage prescribed to patients matches with the dosage based on BMI.

To calculate the optimal cut-off point of BMI that predicts a $10 \%$ discrepancy between dosage based on TBW or ABW.

Materials and Methods Retrospective study January 2003December 2010 performed in a 450-bed tertiary hospital.

Dosage of $15 \mathrm{mg} / \mathrm{TBW}$ was considered except for patients with TBW $>30 \%$ over ideal body weight (IBW). That dose was calculated according to $\mathrm{ABW}$ : $\mathrm{ABW}(\mathrm{kg})=\mathrm{IBW}+0.4(\mathrm{TBW}-\mathrm{IBW})$ as recommended.

Patients included: intravenous amikacin treatment of respiratory tract infections in an extended-interval dosing regimen with therapeutic drug monitoring of amikacin.

Patients excluded: $<18$ years, $\mathrm{ClCr}<60 \mathrm{~mL} / \mathrm{min}$, sepsis, lack of data.

Data collected: demographics, TBW, height, BMI, renal function.

Amikacin levels: fluorescence polarisation immunoassay (TDX, Abbott Lab)

Pharmacokinetic analysis: Bayesian estimation compartmental model (PKS programme)

Statistical analysis: ROC curve.

Results 133 patients $(79.70 \%$ men). Mean $( \pm S D)$ : age: 62.12 years ( \pm 15.48$)$; TBW: $65.52 \mathrm{~kg}$ ( \pm 13.43$)$; height: $166.89 \mathrm{~cm}( \pm 7.44)$; serum creatinine baseline: $0.68( \pm 0.19)$ and $\mathrm{CrCl}: 97.32 \mathrm{~mL} / \mathrm{min}( \pm 34.67)$.

Difference between TBW dose vs. ABW dose (mg) $(\%)$ BMI [<16]: 16.45 vs. $16.45(0 \%)$; BMI[16-18.49]:16.57 vs. $16.57(0 \%)$; BMI[18.5-24.9]:15.28 vs. 15.61(2.2\%); BMI[25-29.9]:12.70 vs. 14.30(11.2\%); BMI[30-34.9]:11.56 vs. 14.34(19.3\%); BMI[35-39.9] and [>40]: 1 patient.

A ROC curve was built to determine the best cut off point of BMI: $26 \mathrm{mg} / \mathrm{m}^{2}$

Difference between recommended dosage and prescribed dosage (mg): BMI $[<16]$ : +1.45; BMI 16-18.49: +1.58; BMI[18.5-24.9] +0.64; BMI[25-29.9]: -0.70; BMI[30-34.9]: -0.66; BMI[35-39.9] and [>40]: 1 patient.

Conclusions Considerable variation between the dosage of amikacin based on TBW and ABW was observed with a reduction of recommended dose in patients with $\mathrm{BMI} \geq 25 \mathrm{~kg} / \mathrm{m}^{2}$ and an overdose in patients with BMI $<24.9 \mathrm{~kg} / \mathrm{m}^{2}$.

A reduction of $10 \%$ or more of the adjusted calculated dose of amikacin was observed in patients with BMI $\geq 26 \mathrm{~kg} / \mathrm{m}^{2}$.

No conflict of interest.

\section{PHC-002 ANALYSIS OF THE INCIDENCE OF POTENTIAL DRUG INTERACTIONS IN HOSPITALISED PATIENTS}

doi:10.1136/ejhpharm-2013-000276.347

M Wieczorek. SPZOZ WSS, Hospital Pharmacy Department, Rybnik, Poland

Background Prescriptions with more than one drug increase the risk of drug-drug interactions, treatment failure, large pharmacological effects and adverse events. 